\title{
Holarchic Psychoinformatics: A Mathematical Ontology for General and Psychological Realities
}

\author{
Suraj Sood ${ }^{1}$, Corinne Lee ${ }^{3}$, Garri Hovhannisyan ${ }^{2}$, Shannon Lee ${ }^{3}$, Garrett Rozier ${ }^{1}$, Antoinette \\ Hadgis $^{3}$, Kristy Sproul ${ }^{3}$, Tyler Higgins ${ }^{3}$, Anna Henson ${ }^{4}$, Michael Shrider ${ }^{3}$, and Monte \\ Hancock $^{6}$ \\ ${ }^{1}$ University of West Georgia, Carrollton, GA, U.S. \\ ${ }^{2}$ Duquesne University, Pittsburgh, PA, U.S. \\ ${ }^{3}$ Sirius Project \\ ${ }^{4}$ Carnegie Melon University, Pittsburgh, U.S. \\ ${ }^{6}$ Digital, Buffalo, U.S. \\ sirius19confegmail.com
}

\begin{abstract}
This article builds upon a formal person-situation framework by offering formalisms for its subcomponents, as well as for reality more generally. More specifically, a system of mathematical formalisms is offered relating the following constructs: holarchy, reality, and psychological reality. Psychological reality is offered a portmanteau in the neologism, "psychologicality". Psychologicality denotes mind and behavior, both of which are subcomponents of Sood's [20] person-situation formula. The mathematics offered is woven into a broader, subjective-objective ontology that is required for any truly representative virtual world.
\end{abstract}

The result from above, when synthesized with informatics, is a novel "holarchic informatics" and more specific holarchic psychoinformatics (HPI). Holarchic psychoinformatics is related with Sood's psychoinformatic complexity (PIC) paradigm.

The aim of this article is to build upon "third force"-i.e., existential-humanistic (E-H) - psychology. We depart from more traditional approaches in defining E-H psychology as the study of the existence of human minds and behaviors as emergent, interdependent properties of people's interactions with situations. This definition results from the enactive person-situation framework as situated within PIC.

Keywords: third-force psychology, person-situation interaction, holarchy, psychoinformatics, ontology

\section{$1 \quad$ Introduction}

What is or are the futures of "third force", i.e. existential-humanistic (E-H) psychology? To answer this question, it must first be asked what E-H psychology's raison d'être is and whether it has been fulfilled. If existential psychologists are interested in the existence of psychological beings and humanistic psychologists focus on human psychology most broadly, then E-H psychologists should be concerned with the existence of humans as psychological beings. Given the broadness of this terrain, it will be granted from the outset that E-H psychology has not yet exhausted its contributive capabilities. 
Indeed - assuming that existence as well as human psychology are equally dynamic as they are staticthere will always be some need for E-H psychology to update its understanding of the existence of humans as psychological beings, as such beings evolve.

What, then, characterizes the existence of humans as psychological beings? Wilber [25] argued for a subjective-objective model of reality—or, for him (as well as this paper's purposes), "holarchy"—of which human psychology is a more recent part. At present, psychological science is most often defined as that of mind and behavior (see, e.g., [14]). Thus, E-H psychology should be concerned more specifically with the existence of human minds and behaviors.

In this paper, contributions to a novel "holarchic-informatic psychology" (that is consonant with, yet distinct from, E-H psychology) are summarized and forwarded. Said contributions include:

1. A feature list intended to facilitate the definition of human beings

2. A holarchic system of formalisms that situates "psychological reality" [17] as its own metaphysical domain, and further delineates the sub-domain's essential subject matter The above contributions are then related to the fields of informatics and psychoinformatics. The result will be a "holarchic psychoinformatics" (HPI) related to the psychoinformatic complexity (PIC) paradigm [20] that unites psychoinformatics with complexity science.

\section{$2 \quad$ Related Work}

Sood [20] posited 18 human features in answering the question of what it means to be human (or, more specifically_people). Sood's features include:

1. Physical - People's bodies are composed of matter. Further, people interact with other physical objects.

2. Biological - People breathe, eat, and drink; and a great many of them have sex and reproduce.

3. Temporal - People are born, they live, and they die; they experience time.

4. Cultural - People are embedded in cultures characterized by unique but shared ways of being.

5. Social-People participate in societies consisting of concrete relations between themselves and others.

6. Economic - People are agents who trade goods and services with one another in marketplaces.

7. Technological - People invent and utilize tools to perform tasks they were previously unable or less able to accomplish.

8. Artistic-People express themselves through the creation of original works such as paintings and songs.

9. Intellectual - People aim to comprehend reality and achieve accurate understandings of it.

10. Moral - People have unique and shared ideas of wrong versus right action.

11. Spiritual - People seek enlightenment, wisdom, and contact with the divine or supernatural via practices such as meditation and prayer.

12. Religious - People worship what they deem as sacred (e.g., God or Gods) through rituals and organized communion.

13. Political - People negotiate and have interests that are in line or at odds with those of others.

14. Athletic - Whether for fitness or organized play, people exercise their bodies and minds. 
15. Professional - People work toward particular goals, including earning money and achieving satisfaction.

16. Recreational - People enjoy leisurely activities such as taking walks and attending parties.

17. Linguistic - People communicate via representational symbol systems characterized by semantics, syntax, and pragmatics.

18. Psychological - People have minds and engage in behaviors. More specifically, they think, feel, have personalities, interact with situations, are motivated, sense, perceive, and experience.

The above list may be considered more relevant to personology than personality, proper. McCrae and Costa [12] discussed "personologists" (p. 81) but did not distinguish such researchers from personality psychologists. Still, given that \#18 above states the core topics of psychological inquiry, one could most reasonably expect E-H psychologists to focus on the sum-total of its items. These psychologists would therefore need to include cognition, affect, personality, situationality, behavior, motivation, sensation, perception, and experience in their ultimate descriptions of who people are, their explanations of how people come to be, and their predictions of whom people are expected to become.

Within psychology, Freud was the pioneer of personality vis-à-vis mind as much as Skinner was the same for behavior [8]. Affect has been addressed by psychologists via the five factor model (FFM) constructs of Extraversion and Neuroticism; cognition was included in Kelly's [9] personal construct and Dweck and Leggett's [3] social-cognitive theories. Lastly, experience, meaning, and motivation have been taken up by third force theorists such as Kelly, Maslow, and Rogers in addition to positive psychologists like Proctor, Tweed, and Morris [15].

Despite the progress summarized above, it remains an open question whether psychologists have fully accounted for both people and their situations. What determines their interaction? The best-established construct that is closest to the former is personality. Situations, on the other hand, have no corresponding construct denoting situationality. It may be partially inferred from this latter fact that psychologists understand personality better than situationality. In part to ameliorate this situation, Sood [20] formalized person-situation interaction in the following manner

$$
F[P, S]=\left[S t_{T, S e}, P c\right]_{M, B}
$$

Where $P$ equals "person", $S$ equals "situation", $S t$ equals "structure", $T$ equals "trait", $S e$ equals "state", $P c$ equals "process", $M$ equals "mind”, and $B$ equals "behavior" [20]. (Traits and states are treated as distinct types of psychological structures.) According to (1), $F[P, S]$ is a whole composed entirely of parts $S t_{M S e}, S t_{B S e}, S t_{M T}, S t_{B T}, P c_{M}$, and $P c_{B}$, which respectively denote "mental states", "behavioral states", "mental traits", "behavioral traits", "mental processes", and "behavioral processes". The kind of person-situation interaction expressed through (1) — which has been formalized to render the construct more applicable within mathematical, theoretical, and computational contexts - is thus distinctly psychological in accommodating mind and behavior (two of psychology's highest-level topics of study).

${ }^{1}$ All sufficiently-similar equations offered hereon are syntactically consistently with Lewin's field theory of behavior [11] and Sood's enactive person-situation formula. Two-letter variable-naming is allowed to the extent that the same is in software program variable declaration, and is particularly necessary in cases of multiple constructs beginning with identical first letters. 
Sood's efforts above represent a start for the formalization of psychological reality. What was left out were formalisms specifically for $M$ and $B$, as well as for the broader reality of which psychology is merely one part. The next section elaborates formulae for a more general metaphysics or ontology.

\section{$3 \quad$ Holarchy and Psychological Reality}

Metaphysics and related formalizations within which to situate holarchy and psychological reality are now laid out. Wilber's metaphysics represents a recent and highly integrative subjective-objective, parts-wholes one - consequently, it will serve as the starting point for this and following sections' formalisms.

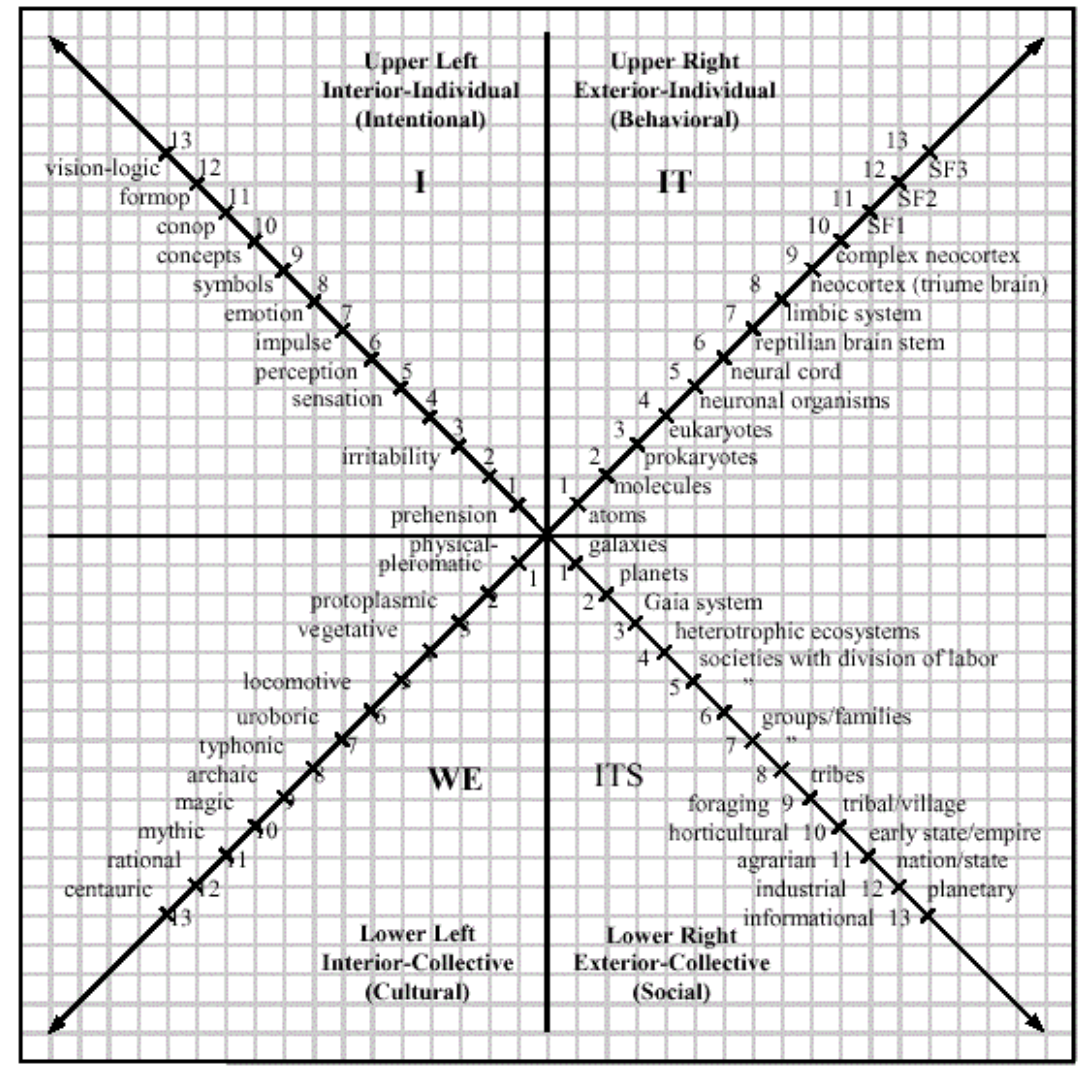

Fig. 1. Wilber's [25] hierarchical four-quadrant metaphysics, consisting of parts-wholes ("holons") of subjective and objective structures and processes (p. 198). 
Holarchy has been used to refer specifically to hierarchical systems composed of holons (e.g., a la Koestler in [10]). Reality, however, consists of both hierarchies and "heterarchies" [24]. The present analysis departs significantly from Wilber and (to a greater extent) Koestler in asserting that reality is and ought to be equally hierarchical-consisting of sets of necessary and sufficient quantitative difference — and heterarchical—consisting of sets whose members share necessary and sufficient qualitative similarity.

Wilber's holarchic subjective-objective model of reality yields the most general possible formula in terms of function $F$

$$
H=F[I(O, S j)]
$$

Where $H$ equals "holarchy", $I$ equals "inter", $O$ equals "objectivity", and $S j$ equals "subjectivity" (such that $I(O)$ and $S j(O)$ stand for "interobjectivity" and "intersubjectivity", respectively). In addition to subjects, objects, and holons, $I(O)$ and $I(S j)$ are each also comprised of forces or processes. Any complete metaphysics must include substance and process: each, in equal quantity.

Table 1. Wilber's holonic, subjective-objective metaphysics (i.e., holarchy).

\begin{tabular}{|c|c|c|}
\hline & Mental being(s) & Behavioral object(s) \\
\hline Holon & $S j: 1$ subject & $O: 1$ object \\
\hline Holons & $I(S j): \geq 2$ subjects & $I(O): \geq 2$ objects \\
\hline
\end{tabular}

Reality can be understood as being made up of physicality, "chemicality", "biologicality", "psychologicality", sociality, "culturality", and spirituality: in essence, all broad domains of existence ${ }^{2}$. Given that holons are the most fundamental units of each of these domains, they enable the theoretical and scientific union of all present areas of such study. Holons may be either subjective (in Wilber's terms, "interior") or objective (for Wilber, "exterior"). A more general and elementary requirement for holons - i.e., that they exist - is further granted.

The next equation follows from what has been said about reality thus far

$$
R=F[P h, C, B l, \psi, S c, C l, S p]
$$

\footnotetext{
${ }^{2}$ One could include other domains in addition to those offered herein. Such possible additions include temporality, "economicality", "politicality", "technologicality", and "religiality". The included sub-domains have been chosen over candidates such as these purely given the novelty of the approach undertaken and in the interest of ensuring tractability.
} 
Where $R$ equals "reality", $P h$ equals "physicality”, $C$ equals "chemicality”, $B l$ equals "biologicality”, $\psi$ equals "psychologicality", $S c$ equals "sociality", $C l$ equals "culturality", and $S p$ equals "spirituality". ${ }^{3}$ Given the massive (and potentially greater) inclusivity of the right half of (3), $R$ is essentially equal to $H$. Thus, (4) is stipulated

$$
H=R
$$

Via (3), (4), and the transitive property, (5) results

$$
H=F[P h, C, B l, \psi, S c, C l, S p]
$$

And lastly_ as a result of (3), (4), and (5), and again applying the transitive property - (6) follows

$$
F[I(O), I(S j)]=F[P h, C, B l, \psi, S c, C l, S p]
$$

The order in which (6)'s righthand elements have been listed is deliberate. Specifically, $P h$ is characterized by the greatest degree of (inter)objectivity and the lowest amount of (inter)subjectivity, whereas the inverse of this holds true for $S p . \psi$ may be considered unique in this particular scheme for being equally made up of both $I(O)$ (including exterior behavior) and $I(S j)$ (interior mind, i.e. "mentality").

So much for highest-level formalizations of holarchy and reality. Equations are next proposed for $\psi$ (and, subsequently, are defined and related to (6)).

\subsection{The $\psi$ Equation}

Both mind and behavior must be equally and exhaustively incorporated into a completed understanding of psychologicality - or "psychological reality", a construct whose absence of understanding has been lamented by Robinson ([17], p. 191). The general form of such an equation is as follows

$$
\psi=F[M, B]
$$

Where (as they did in (1)) $M$ equals mind and $B$ equals behavior. Any of (7)'s values can be either described-i.e., via first-person reports of interior states/traits and processes, or via third-person descriptions of exterior ones - or measured (e.g., through laboratory experimentation or survey analysis). Ready examples of disciplinary relevance for $M$ are fields like augmented cognition, cognitive science, computer science, cognitive neuroscience, cognitive psychology, and clinical and positive psychologies. $B$ may be understood as being centrally relevant to any more physical or mechanistic (i.e., objective) domain including physiology, neurology, anatomy, computer science, and

\footnotetext{
${ }^{3}$ Why are the neologisms represented via (3) necessary? Existing words like "psychology" are potentially ambiguous, referring either to particular beings—e.g., primates — or the field of psychology (including its subdisciplines, e.g. humanistic psychology). Hitherto, no analyses have utilized terms like psychologicality to refer to the totality of psychological reality: the general formalization of this is undertaken via (7)).
} 
behavioral psychology. Therefore, (7) accommodates a multimethodological (viz., philosophicalqualitative or theoretical-quantitative) analysis. ${ }^{4}$

\section{2 $\quad M$ and $B: \psi$ Sub-Equations}

For the $M$ portion of $\psi,(8)$ is asserted

$$
\left.M=F\left[(A, C, M v)_{(U-,} S b-\right) C s\right]
$$

Where $A$ equals "affect", $C$ equals "cognition", $M v$ equals "motivation", $U-$ equals "un-", $S b-$ equals "sub-", and Cs equals "consciousness". According to the right portion of (7)'s subscript, each of these elementary mental phenomena may be either unconscious, subconscious, or conscious. (8) yields the following nine constructs: "unconscious affect", "subconscious affect", "conscious affect"; "unconscious cognition", "subconscious cognition", "conscious cognition"; and "unconscious motivation", "subconscious motivation", and "conscious motivation". 5

Formula (8) draws from Freud's topographical model of mind [5] on one hand-where mental content passes between the unconscious and conscious sub-minds via the intermediary subconsciousand Revelle's recent attempt to synthesize Plato's tripartite model of mind (consisting of precursors for affect, cognition, and motivation) into a formal personality framework [16].

For the $B$ sub-portion of $\psi,(9)$ is added

$$
B=F[S m, R p]
$$

Where $S m$ equals "stimulus" and $R p$ equals "response". Equation (9) draws largely from Skinner's $S-R$ theory [19], wherein behavior was put forth as consisting of any environmental stimulus and a necessary behavioral response. $B$ is distinct from $M$ in that the latter consists of interactive interior components rather than exterior ones. The present neuro-cognitive paradigm represents a modern-day reframing of $\psi$ in terms of specifiable correlations demonstrated to exist between neural mechanicse.g., "all-or-nothing" neural firing - and cognitive processes (e.g., reasoning). $M$ and $B$ 's subcomponents may each be interpreted comparatively more subjectively (e.g., via self-report and "other-report") or explained (e.g., in terms of mental or behavioral mechanisms). Thus, they are equally amenable to a "mixed methods" (see, e.g., [2, 22]) psychological approach.

Psychologicality has evolved in many present-day societies (particularly North-Western ones) to become more informational. In light of this, an explicitly psychoinformatic view of humans such as that offered by Sood [20] will be necessary.

\section{$4 \quad$ Holarchic Psychoinformatics}

\footnotetext{
${ }^{4}$ Philosophical and theoretical approaches are distinguished in that the former are characterized as being more exploratory or question-focused. Works of the latter kind are comparatively more explanatory or answer-focused. Given the more open-ended nature of qualitative inquiry and the definitive nature of quantitative, the former here is arguably closer in spirit to philosophical approaches just as theoretical and quantitative are with one another.

${ }^{5}$ As it relates with (1), each of (8)'s primitive psychological values may be mental structures-i.e., states or traits (e.g., "conscious affective states" like palpable, passing moods)—or processes (e.g., becoming motivated to carry out a given task).
} 
How is the holarchic paradigm related to informatics? Gruska [26] described the latter discipline's "main task" as being "to discover, explore and exploit in depth the laws, limitations, paradigms, concepts, models, theories, structures and processes of both natural and virtual information processing worlds and to explore their phenomena as well as their interrelations, impacts and utilization" (p. 6). The more specific area of psychoinformatics was defined by Yarkoni [27] as "an emerging discipline that uses tools and techniques from the computer and information sciences to improve the acquisition, organization, and synthesis of psychological data" (p. 391).

Sood [20] introduced the term "psychoinformatic complexity" (PIC) to denote a paradigm marrying psychoinformatics (as defined above), and complexity science as defined by Bar-Yam [28]. Hancock et al.'s work [7] - which utilized an algorithm of computational complexity $O\left(N^{2}\right)$ to infer, characterize, and visualize the emotional context arising from online social discourse-was cited as fulfilling PIC's necessary and sufficient criteria for membership. Said criteria included conformation to Yarkoni's psychoinformatic definition, as well as to Bar-Yam's framing of complexity science being centrally interested in systemic emergence and interdependence (p. 25).

A holarchic informatics would synthesize holarchy as formalized in this paper with Gruska's informatics. Holarchy has been defined as any subjective-objective reality-including at least the subdomains $\{P h, \ldots S p\}$ - consisting equally of hierarchical and heterarchical, holonic (part-whole) systems. A holarchic informatics is to be interested in information-processing within any of holarchy's subdomains.

Lastly, a holarchic psychoinformatics (HPI) would represent a paradigm akin to PIC, but with emphasis on holarchy rather than complexity. Such a framework closely resembles Henriques' [8] evolutionary epistemology in which matter gave way to life; life gave way to mind; mind gave way to society; and, lastly, society gave way to culture. The difference lies specifically in the psycho-prefix of psychoinformatics, which specifies the domain of mind (and behavior) as the central HPI focus for any E-H psychologist. 


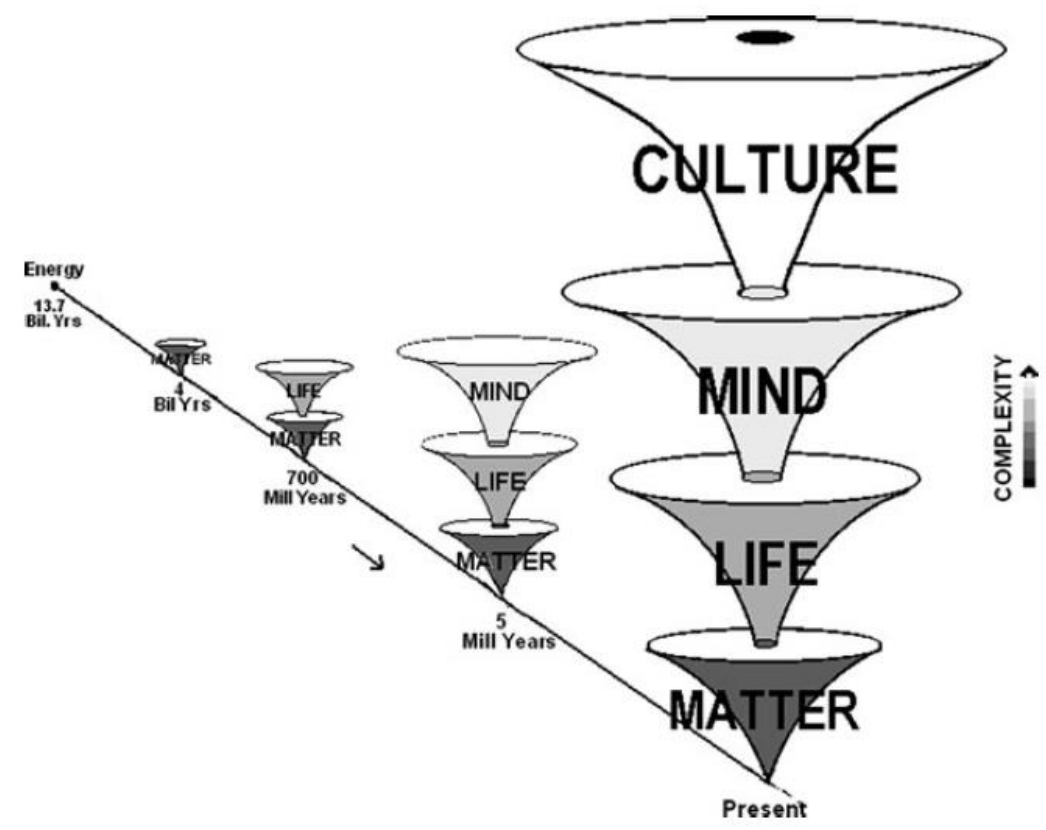

Fig. 2. Henriques" "Tree of Knowledge" system (p. 14).

Information-processing is present at all levels of Henriques' system, thus making it a PIC and HPI one at the level of mind in particular. One could thus speak further of a "holarchic psychoinformatic complexity" (HPIC) super-paradigm, though this is beyond the scope of the present article.

\section{Conclusion}

In this article, a holarchic-informatic view of psychological reality has been elaborated. If E-H psychology's broadest goal is to understand the existence of humans as psychological beings, then it needs to account for both its subjective (i.e., mental) and objective (i.e., behavioral) aspects and forces. It additionally needs to facilitate the more integrated view of person-situation interaction. Finally, E-H psychology should come to terms with the modern-day human's increasing technologization [1]. Thus, one may speak of a holarchic psychoinformatics (HPI) as greatly complementing the E-H project referred to throughout this article. Holarchy specifies both general and psychological realities as being complex and multifaceted, being composed of holons rather than exhaustibly derivable from their parts alone. It further leaves more to the E-H psychologist who is interested in a broader conception of mind as consisting of unconscious-to-conscious affect and motivation in addition to cognition.

The psychoinformatic approach is becoming and will become increasingly more necessary to adopt in understanding the existence of humans as psychological beings. If E-H psychology becomes more holarchic in incorporating both mental and behavioral aspects and forces while recognizing the human's 
increasing virtuality, then it would go further than any unifying theoretical or scientific effort has yet to with respect to modern-day psychological reality.

Acknowledgments. The first author thanks Drs. Christine Simmonds-Moore and John Lloyd Roberts for enabling the opportunities to develop this article.

\section{References}

1. Aanstoos, C.: Humanistic psychology in dialogue with cognitive science and technological culture. In: Schneider, K., Pierson, J., Bugental, J. (eds.) The Handbook of Humanistic Psychology: Theory, Research, and Practice, pp. 348-363. Sage Publications, Los Angeles (2015).

2. Creswell, J.W.: Research design: Qualitative, Quantitative, and Mixed Method Approaches, $3^{\text {rd }}$ edn. Sage Publications, Thousand Oaks (2009).

3. Dweck, C., Leggett, E.: A social-cognitive approach to motivation and personality. Psychol. Rev. 95(2), 256-273 (1988).

4. Fleeson, W., Noftle, E.: The end of the person-situation debate: An emerging synthesis in the answer to the consistency question. Soc. Personal. Psychol. Compass 2(4), 1667-1684 (2008).

5. Freud, S.: The Interpretation of Dreams. (J. Strachey, Trans.) Basic Books, New York (1955). (Original Work Published in 1899)

6. Giordano, P. J.: Individual personality is best understood as process, not structure: A Confucian-inspired perspective. Cult. Psychol. 0(0), 1-17 (2016).

7. Hancock, M., Rajwani, S., Lo, C., Sood, S., Kresses, E., Bleasdale, C., Dunkel, N., Do, E., Rees, G., Steirs, J., Romero, C., Strohschein, D., Powell, K., French, R., Fedosenko, N., Casimir, C.: Field-theoretic modeling method for emotional context in social media: Theory and case study. In: Schmorrow, D., Fidopiastis, C. (eds.) Augmented Cognition 2015. LNCS, vol. 9183, pp. 418-425. Springer, Cham (2015).

8. Henriques, G.: A New Unified Theory of Psychology. Springer, New York (2011).

9. Kelly, G.: Clinical Psychology and Personality: The Selected Papers of George Kelly. John Wiley \& Sons, Inc., New York (1969).

10. Koestler, A.: The Ghost in the Machine. The Macmillan Company, New York (1967).

11. Lewin, K.: Field theory and experiment in social psychology: Concept and methods, Am. J. Sociol. 44, 868-896 (1939).

12. McCrae, R., Costa, P.: Validation of the five-factor model of personality across instruments and observers. J. Pers. Soc. Psychol. 51(1), 81-90 (1987).

13. Neumann, S., Sood, S., Hollander, M., Wan, F., Ahmed, A., Hancock, M.: Using bots in strategizing group compositions to improve decision-making processes. Lecture Notes in Comput. Sci. 10916, 305-325 (2018).

14. Passer, M. W., Smith, R. E.: Psychology: The Science of Mind and Behavior, $2^{\text {nd }}$ edn. McGraw-Hill, New York (2004).

15. Proctor, C., Tweed, R., Morris, D.: The Rogerian fully functioning person: A positive psychology perspective. J. Humanist. Psychol. 56(5), 1-28 (2015).

16. Revelle, W.: Integrating personality, cognition, and emotion: Putting the dots together? (2011). https://www.personality-project.org/revelle/publications/BPSP-revelle.pdf. Accessed 9 Jan 2019 
17. Robinson, D.N.: Theoretical psychology: What is it and who needs it? Theory Psychol. 17(2), 191-198 (2007).

18. Rogers, C.: The concept of the fully functioning person. Psychotherapy: Theory, Res. \& Pract. 1(1), 17-26 (1963).

19. Skinner, B. F.: Generic nature of the concepts of stimulus and response. J. Gen. Psychol., 1240-1265 (1935).

20. Sood, S.: The psychoinformatic complexity of humanness and person-situation interaction. https://psyarxiv.com/fnjte. Accessed 28 Nov 2018

21. Sood, S.: Global problem-solving and ethics: A theoretical and practical analysis. Int. J. Environ. Issues 17(4), 322-339 (2018).

22. Tashakkori, A., Teddlie, C., Sines, M.: Utilizing mixed methods in psychological research. In: Weiner, I., Schinka, J., Velicer W. (eds.) Handbook of Psychology, Vol. 2: Research Methods in Psychology, pp. 428-450. John Wiley \& Sons, Hoboken (2012).

23. Varela., F., Thompson, E., Rosch, E.: The Embodied Mind: Cognitive Science and Human Experience. The MIT Press, Cambridge, MA (1991).

24. Von Goldammer, E., Paul, J., Newbury, J.: Heterarchy-hierarchy: Two complementary categories of description (2003). http://vordenker.de/heterarchy/a_heterarchy-e.pdf. Accessed 10 Jan 2019

25. Wilber, K.: Sex, Ecology, Spirituality: The Spirit of Evolution. Shambhala Publications, Boston (2000).

26. Gruska, J.: A perception of informatics, https://westga.view.usg.edu/d21/le/content/1411076/viewContent/24151440/View. Accessed 21 Jun 2018

27. Yarkoni, T.: Psychoinformatics: New horizons at the interface of the psychological and computing sciences. Current Directions in Psychological Science 21, 391-397 (2012).

28. Bar-Yam, Y.: Making things work: Solving complex problems in a complex world. NECSI/Knowledge Press, Cambridge, MA (2004). 